\title{
Surveillance of influenza in Iceland during the 2009 pandemic
}

G Sigmundsdottir (gudrun@landlaeknir.is)¹, T Gudnason¹, ö ólafsson², G E Baldvinsdóttir³, A Atladottir ${ }^{1}$, A Löve³, L Danon4, H Briem ${ }^{1}$

1. Centre for Health Security and Communicable Disease Control, Directorate of Health in Iceland, Seltjarnarnes, Iceland

2. Public Health Sciences, University of Iceland, Reykjavík, Iceland

3. Department of Virology, Landspitali University Hospital, University of Iceland, Reykjavík, Iceland

4. Department of Biological Sciences, University of Warwick, Warwick, United Kingdom

Citation style for this article:

Sigmundsdottir G, Gudnason T, Ólafsson Ö, Baldvinsdóttir GE, Atladottir A, Löve A, Danon L, Briem H. Surveillance of influenza in Iceland during the 2009 pandemic. Euro Surveill. 2010;15(49):pii=19742. Available online: http://www.eurosurveillance.org/ViewArticle.aspx?Articleld=19742

Article published on 9 December 2010

In a pandemic setting, surveillance is essential to monitor the spread of the disease and assess its impact. Appropriate mitigation and healthcare preparedness strategies depend on fast and accurate epidemic surveillance data. During the 2009 influenza $A\left(\mathrm{H}_{1} \mathrm{~N}_{1}\right)$ pandemic, rapid improvements in influenza surveillance were made in Iceland. Here, we describe the improvements made in influenza surveillance during the pandemic, which could also be of great value in outbreaks caused by other pathogens. Following the raised level of pandemic influenza alert in April 2009, influenza surveillance was intensified. A comprehensive automatic surveillance system for influenza-like illness was developed, surveillance of influenza-related deaths was established and laboratory surveillance for influenza was strengthened. School absenteeism reports were also collected and compared with results from the automatic surveillance system. The first case of 2009 pandemic influenza $A\left(\mathrm{H}_{1} \mathrm{~N}_{1}\right)$ was diagnosed in Iceland in May 2009, but sustained community transmission was not confirmed until mid-August. The pandemic virus circulated during the summer and early autumn before an abrupt increase in the number of cases was observed in October. There were large outbreaks in elementary schools for children aged 6-15 years throughout the country that peaked in late October. School absenteeism reports from all elementary schools in Iceland gave a similar epidemiological curve as that from data from the healthcare system. Estimates of the proportion of the population infected with the pandemic virus ranged from $10 \%$ to $22 \%$. This study shows how the sudden need for improved surveillance in the pandemic led to rapid improvements in data collection in Iceland. This reporting system will be improved upon and expanded to include other notifiable diseases, to ensure accurate and timely collection of epidemiological data.

\section{Introduction}

The first reports of 2009 pandemic influenza $A\left(\mathrm{H}_{1} \mathrm{~N}_{1}\right)$ in humans in the United States and Mexico appeared in April 2009 [1]. Initial descriptions of the outbreak in Mexico were alarming, with severe cases of pneumonia and high mortality in previously healthy young adults being reported [1]. On 27 April 2009, the World Health Organization (WHO) raised the level of pandemic influenza alert from phase three to four and two days later from phase four to five $[2,3]$. Countries were encouraged to activate their pandemic preparedness plans and remain on high alert for unusual outbreaks of influenza-like illness and severe pneumonia. In a pandemic, both clinical and epidemiological data are essential in attempts to assess the severity of the illness. The allocation of healthcare resources and choice of appropriate intervention strategies also rely on accurate and timely surveillance data. Such data are essential in identifying groups at risk of severe illness and who should be prioritised in vaccination strategies. Surveillance is also needed to evaluate the impact of different interventions. Heightened surveillance was therefore a high priority during the pandemic in order to detect the first cases and monitor the spread of the disease.

Conventional surveillance methods for influenza are mostly based on laboratory surveillance and sentinel surveillance of influenza-like illness (ILI), but interest in mortality surveillance has increased during the last decade $[4,5]$. Unconventional surveillance methods, such as school absenteeism, syndromic surveillance and mobile phone surveillance, have also been used but these methods require further validation [6-8]. All elementary schools for children aged 6-15 years in Iceland enter information on school absenteeism into a common database, but these data have not been analysed for epidemiological purposes so far [9].

There were differences in healthcare services, surveillance and interventions between European countries during the 2009 pandemic. Reports from individual countries on the pandemic are therefore crucial to compare experiences, share knowledge and maximise the lessons learned after the pandemic. In this article we report the changes made in the surveillance of influenza in Iceland and describe the data collected during the pandemic. 
Surveillance systems in Iceland

Surveillance of influenza-like illness

In April 2009 surveillance of ILI in Iceland was based on monthly paper-based reporting of aggregated data from primary healthcare centres to the Centre for Health Security and Communicable Disease Control (CHS-CDC). After WHO initially raised the pandemic alert level, Icelandic legislation was changed allowing personal, identifiable information to be collected for each case. Simultaneously, an online automatic system for immediate reporting of ILI and cases with laboratory-confirmed influenza to the CHS-CDC was developed, using the same software used for electronic patient records in primary health care and hospitals in Iceland [10].

The current International Classification of Diseases (ICD-10) for standard diagnostic classification and International Classification of Primary Care (ICPC-2) for standard classification of a patient's reason for encounter were used to identify ILI and confirmed influenza cases for automatic online reporting in the system $[11,12]$. The following ICD-10 codes were used: J10, J10.0 J10.1, J10.8, J11, J11.0, J11.1, J11.8 and U05.9; the ICPC-2 code used was R80. Whenever physicians suspected ILI or diagnosed confirmed influenza they were asked to use the appropriate ICD-10 code in their reporting. After the physician confirmed his record for the patient visit in the electronic patient journal cases with ICD-10 codes for ILI and confirmed influenza were automatically selected and automatically reported within 24 hours via a closed electronic network to the CHC-CDC comprising all healthcare centres and hospitals in Iceland. The data collected for each case included: name, personal identification number, date of birth, place of residence, date of visit to the healthcare centre or hospital, patient's age, sex, which healthcare service the case attended, medical licence number and name of attending physician, the ICD-10 code and the ICPC code. Patients registered with ICD-10 codes for the most common acute respiratory infections (ARI) were also reported automatically and online in the same way as the influenza and ILI cases. Unlike sentinel systems, the automatic reporting system allowed data to be collected from each and every primary healthcare centre and hospital emergency room, thus capturing the vast majority of all diagnosed cases.

The European case definitions for ILI, confirmed cases of seasonal influenza and confirmed cases of

\section{FIGURE 1}

Weekly number of reported cases of influenza-like illness by sex, Iceland, 1 July to 31 December 2009 (n=9,887)

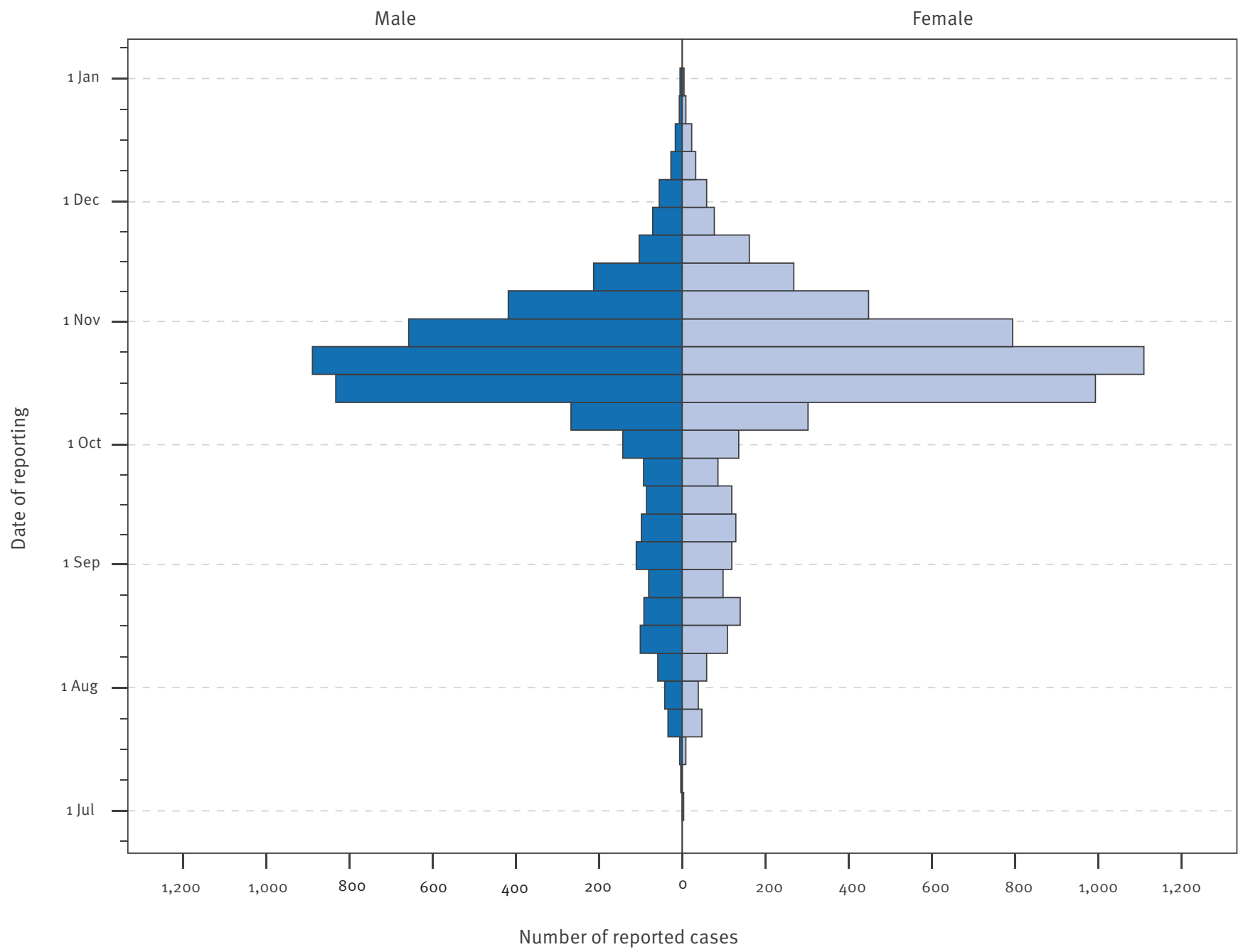


2009 pandemic influenza $\mathrm{A}\left(\mathrm{H}_{1} \mathrm{~N}_{1}\right)$ were used and the selected ICD-10 and ICPC-2 codes were recorded by the physicians [13-14]. In mid-June, when the system was in place, it was also possible to gather data retrospectively from 1 April 2009.

\section{FIGURE 2}

Age-specific incidence of reported influenza-like illness cases by sex, Iceland, 1 July to 31 December 2009

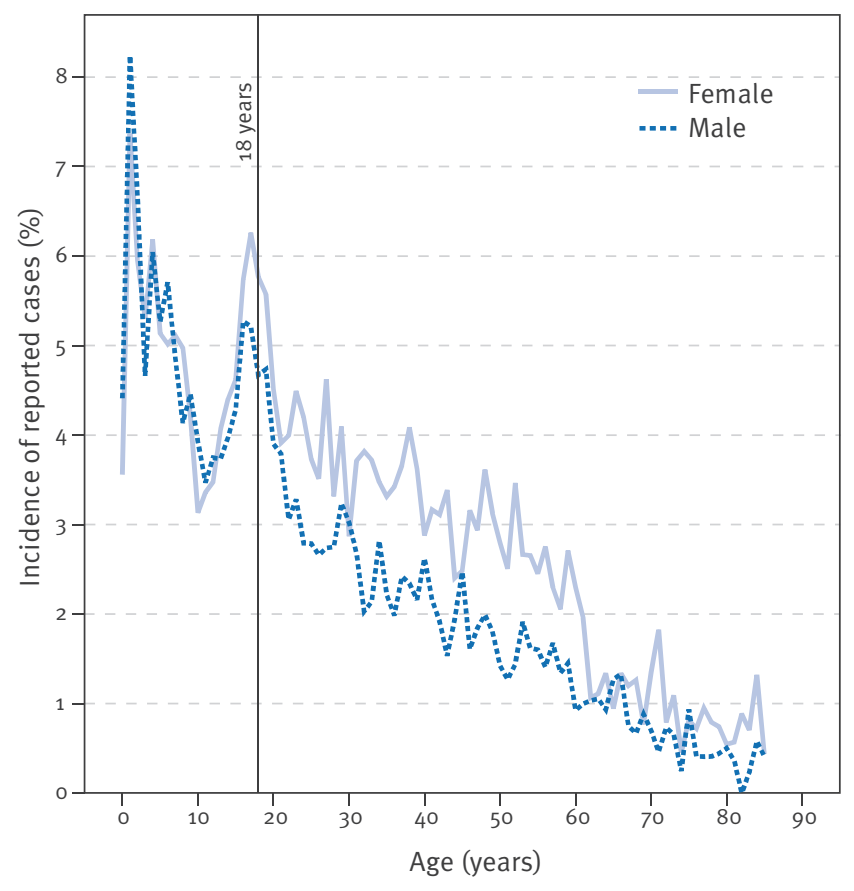

Laboratory surveillance

The Department of Virology at the Landspitali University Hospital in Reykjavik is the sole diagnostic laboratory for influenza in the country. The laboratory received respiratory samples from the nasopharynx and/or throat that were collected from patients with ILI by physicians in primary healthcare centres and at hospitals.

Influenza was diagnosed by real-time polymerase chain reaction (PCR) according to a recommended protocol from the United States Center for Disease Control and Prevention (CDC) [15]. Clinical information and the country of infection were collected on confirmed cases both at the laboratory and at the CHS-CDC. The weekly number of tested respiratory samples and personal information on confirmed cases was reported to the CHS-CDC.

\section{Surveillance of school absenteeism}

All elementary schools in Iceland routinely enter information on school absenteeism for schoolchildren aged 6-15 years into a central database maintained by the information technology company Mentor ehf in Reykjavik [9]. School absence was recorded as the number of days absent; comparable data were available for 2007, 2008 and 2009.

\section{Mortality surveillance}

Mortality data are collected by the National Registry and sent to the CHS-CDC routinely on a weekly basis. The data included the name, personal identification number, date of birth, place of residence and date

\section{FIGURE 3}

Proportional number of reported influenza-like illness cases by age group, Iceland, July to December 2009

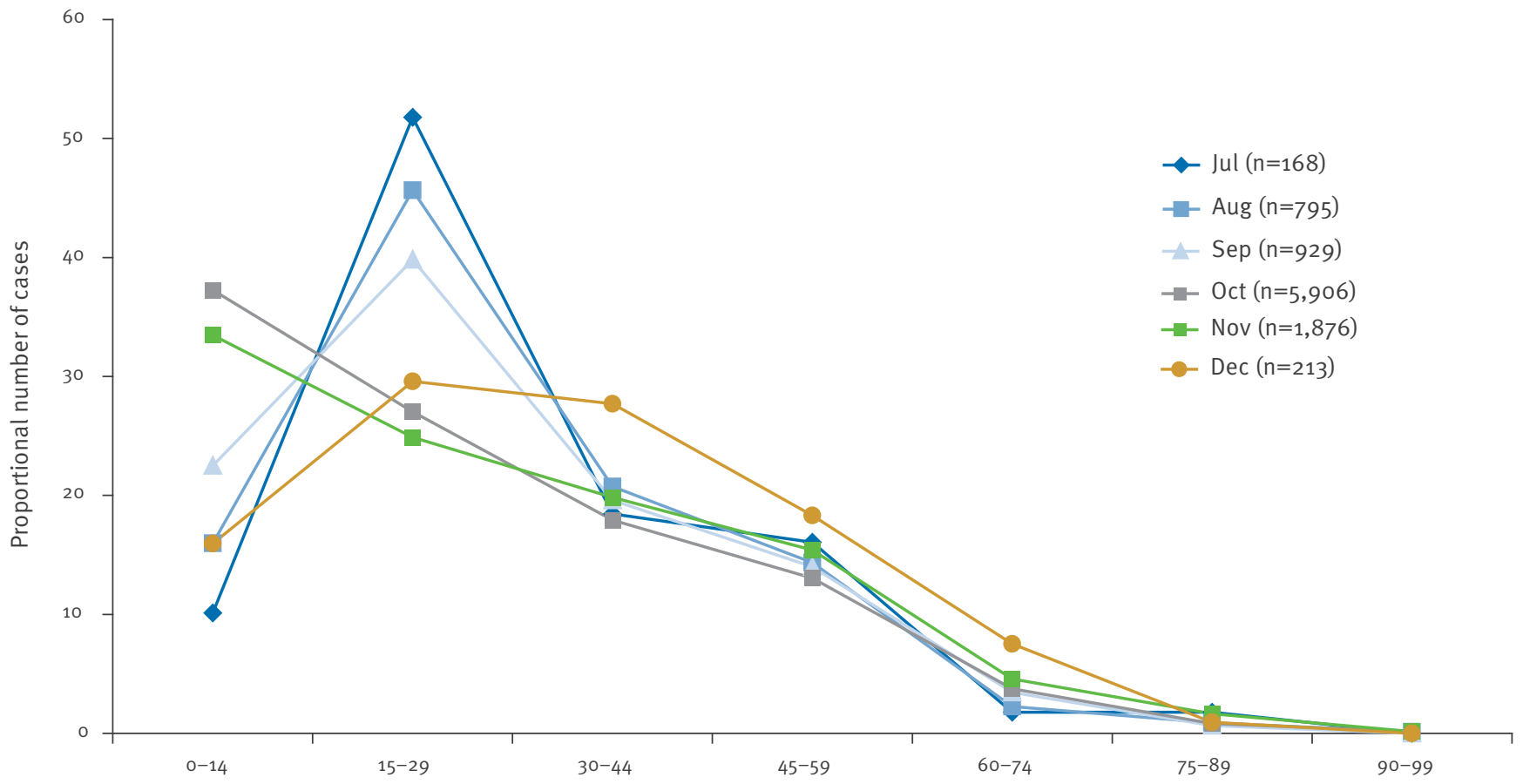

Age group (years) 
of death for each individual. A temporary system for surveillance of patients with ILI and confirmed pandemic influenza admitted to hospital was developed within all hospitals and these cases and deaths in this group were reported immediately to the CHS-CDC. Unexpected deaths in the community in patients with ILI or confirmed pandemic influenza were also to be reported by the physicians to the CHS-CDC.

\section{Data analysis}

\section{Estimated number of infections}

in the community

The percentage of positive laboratory samples was used as an estimate of the proportion of ILI cases in

\section{FIGURE 4}

Cumulative number of reported ILI cases as a proportion of the total number of ILI cases by postal district, Iceland, 1 July to 31 December 2009

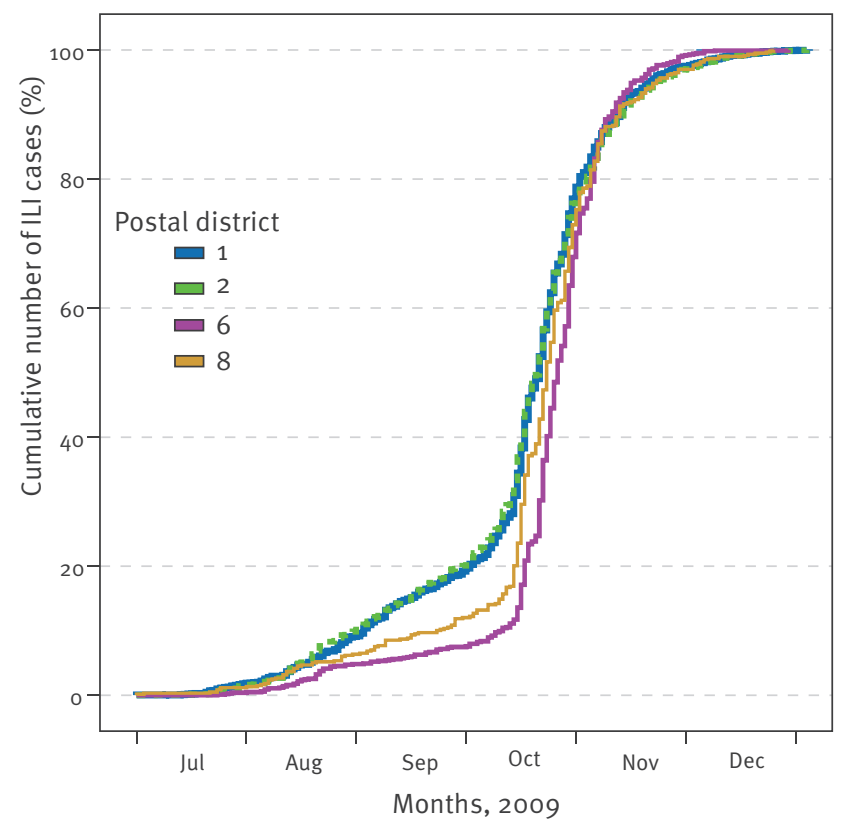

ILI: influenza-like illness.

\section{TABLE}

Reported cases of influenza-like illness by region, Iceland, July to December $2009(n=9,887)$

\begin{tabular}{|l|c|c|c|}
\hline \multirow{2}{*}{ Region } & Postal district & $\begin{array}{c}\text { Number of re- } \\
\text { ported ILI cases }\end{array}$ & Median time $^{\mathrm{a}}$ \\
\hline \multirow{2}{*}{ West Iceland } & 1 & 3,643 & 19 Oct \\
\cline { 2 - 4 } & 2 & 3,019 & 19 Oct \\
\hline West fjords & 3 & 404 & 22 Oct \\
\hline North West & 5 & 109 & 21 Oct \\
\hline North East & 6 & 340 & 27 Oct \\
\hline East Iceland & 7 & 1,016 & 24 Oct \\
\hline South Iceland & 8 & 466 & 22 Oct \\
\hline Westman Islands & 9 & 598 & 21 Oct \\
\hline Unknown & Missing & $\mathbf{8 0}$ & 27 Oct \\
\hline Total & $\mathbf{1 - 9}$ & $\mathbf{9 , 8 8 7}$ & 20 Oct \\
\hline
\end{tabular}

ILI: influenza-like illness.

a The date (in 2009) when half of the ILI cases were reported in the postal district. the community with pandemic influenza. To estimate the total number of infected individuals in the community, we therefore multiplied the weekly number of reported ILI cases by the weekly percentage of laboratory samples confirmed positive for pandemic influenza and summed over the course of the pandemic.

The denominators used in this study were mid-2009 demographic data from the Icelandic Population Registry, according to age, sex and place of residence, as appropriate.

\section{Surveillance data}

Influenza-like illness

Throughout May and June 2009, few cases of ILI and confirmed pandemic influenza were reported. An increase in the number of laboratory-confirmed cases of pandemic influenza was observed from mid-July, when there was a simultaneous absence of confirmed seasonal influenza. Cases of ILI reported from 1 July 2009 onwards were therefore considered to represent the illness caused by pandemic influenza.

From 1 July to 31 December 2009 a total of 9,887 cases of ILI were reported, of whom 5,372 (54\%) were female and $4,515(46 \%)$ were male. The number of cases increased slowly from mid-July to the end of August and fell slightly in mid-September (Figure 1). A sharp increase was observed in October: the number of cases peaked later that month, followed by a rapid decrease. Only sporadic ILI cases were reported in late December.

The incidence of ILI was highest in children and young adults and decreased with age, as shown in Figure 2. ILI incidence was similar in both sexes in people aged under 18 years. However, in people over 60 years, the incidence was higher in women $(p=0.003)$, but the largest difference by sex was observed in people aged 18-59 years, with incidence again higher in females (p<0.001) (Figure 2).

Figure 3 shows how the age of the reported ILI cases changed with time. In July to September 2009, most cases were reported in the 15-30 years age group, but a sudden change was observed in October, when the majority of cases were aged from o to 15 years (Figure 3).

Reported ILI cases were categorised by the postcode of their place of residence. The cumulative number of reported cases over time is given for the four most populated postal districts in the south-west, north and south of the country (Figure 4). There was some indication of spatial dispersal in late September 2009; the number of reported cases increased earlier in the south-west postal districts 1 and 2, followed by an abrupt increase in mid-October in all districts at the same time. The overall number of cases peaked shortly after mid-October (Figure 1, Table).

Data from the surveillance of ARI from the same automatic online system showed similar trends over time as 
the ILI cases, with a peak in early to mid-October 2009 (week 41) (unpublished data).

\section{Laboratory-confirmed cases}

of pandemic influenza

From May to mid-August 2009, physicians were encouraged by the chief epidemiologist to take samples from patients with ILI. The first case of pandemic influenza in the country was laboratory confirmed on 19 May 2009. Three confirmed cases were identified in June, but in late July and August (week 30 to 33) an increase in the number of cases was observed. The first cases in May and June acquired the infection abroad or their infection was domestically acquired with known connection to another confirmed case. The proportion of domestic cases with no known connection to other confirmed cases increased rapidly in July and August. In mid-August (week 33), sustained transmission of infection was confirmed in Iceland and decreased sampling was recommended by the Chief Epidemiologist. From that point on, diagnosis of influenza was based on the physician's clinical examination, and samples were to be obtained only from patients with severe illness or increased risk of serious illness.

\section{FIGURE 5}

Number of respiratory samples and proportion positive for 2009 pandemic influenza A(H1N1), Iceland, 29 June to 27

December 2009

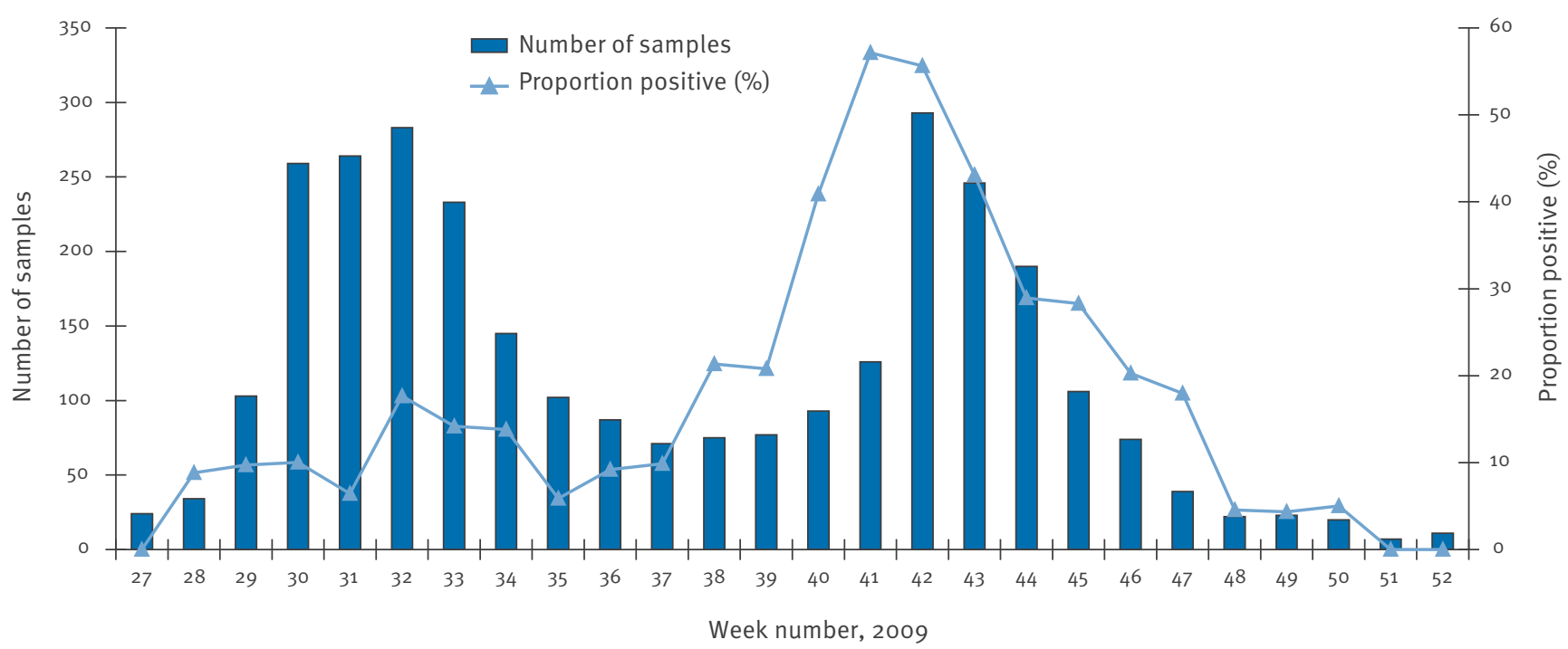

\section{FIGURE 6}

School absenteeism in elementary schools counted in number of days missed, Iceland, weeks 33-52 in 2007-2009

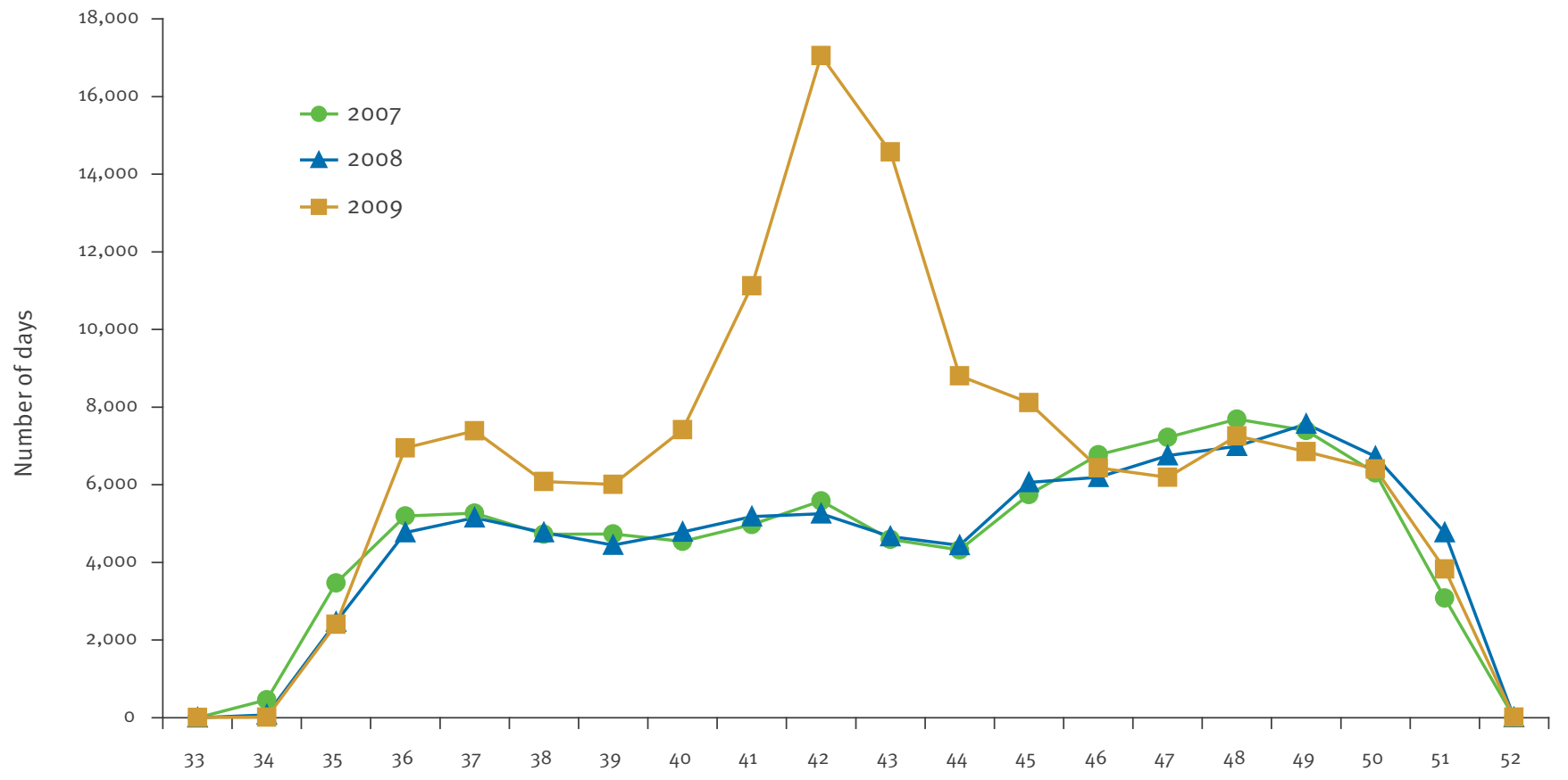

Week number, 2009 
Following this recommendation, there was a decrease in late August 2009 (week 34 and 35) in the number of respiratory samples collected, with a concomitant decrease in the number of laboratory-confirmed cases (Figure 5). From the end of June to the end of December (weeks 27-53), 3,011 samples were collected, of which $702(23 \%)$ tested positive for the pandemic virus. The number of samples and the percentage of samples positive increased in late September (week 40) and peaked in mid-October (week 42), when 293 samples were collected, $56 \%$ of which tested positive. These patterns were consistent with the changes observed in the number of reported ILI cases.

Pandemic influenza was laboratory confirmed in people living in all regions of the country. The age distribution of cases with laboratory-confirmed infections was the same as that observed for reported ILI cases (unpublished data).

\section{School absenteeism}

In September 2009 (week 40), shortly after the school year started, an increase in school absenteeism was observed, compared with the levels at that time in the previous two years (Figure 6). A sharp increase was observed in October 2009, compared with the same period in the previous two years, with a high peak in mid-October (week 42) (Figure 6). In late October and November (week 43 to 46), there was a rapid fall in school absenteeism and from mid-November to the end of December it was similar to that seen in the two previous years.

\section{Mortality levels}

No increase in overall mortality was observed from September to December 2009, according to data from the National Registry. Two persons with laboratoryconfirmed pandemic influenza died during this time: an 18-year-old woman and an 81-year-old man who both had underlying conditions.

\section{Estimated number of pandemic influenza infections in the community}

A total of 3,336 cases were expected to be positive if all ILI cases were tested. This is a lower bound estimate since, in the latter part of the epidemic; tests were performed primarily on severe cases that could be caused by complications, rather than influenza. According to previous studies, approximately $10 \%$ of symptomatic influenza cases occur in the community for each ILI case detected by the surveillance system $[16,17]$. The expected number of symptomatic cases would therefore be 33,368 or $10.4 \%$ of the total population $(n=319.246)$. A large number of asymptomatic infections are also expected to have occurred. A more detailed model has been used to estimate the number of 2009 pandemic influenza infections in the United Kingdom more closely [18], but such modelling is beyond the scope of our study.

\section{Discussion}

This article summarises the surveillance and epidemiology of the pandemic influenza in Iceland in 2009, showing how rapid improvements in influenza surveillance were feasible by connecting the existing structure in the healthcare system for patient records to electronic surveillance system for reporting ILI cases. This system does not require any additional input from physicians, enabling comprehensive data from the entire country to be collected with near real-time information on the geographical spread, age and sex of ILI cases.

The initial increase in the number of ILI cases was first observed in the western regions of the country, with eastern regions following approximately one week later; the peak of ILI activity showed a similar delay (Figure 2 and Table). A west-to-east spread has been described in four of eight influenza seasons from 1999 to 2007 in Europe [19]. The most likely explanation for the direction of spread of the epidemic in Iceland is that the densely populated area of the capital Reykjavik in the south-west corner of Iceland provides ample opportunities for the spread of the pandemic virus; most foreign travel, whether for business or leisure, begins or ends in Reykjavik.

The difference in the number of reported ILI cases by sex in our data could be due to females being more prone to the disease than men, but this hypothesis is not supported by previous studies, with the exception of increased risk of severe illness in pregnant women [20]. An alternative explanation could be that females contact physicians more often than males. The initiative to contact the physician for children and older people who are ill often comes from parents or other close relatives without regard the patient's sex, which could explain equal ILI reporting rate by sex for children and minor sex differences in the rates of reporting of older people. Adults from 18 to 60 years, however, decide themselves when to contact the physician and the differences between males and females observed in that age group in our data probably reflect more frequent visits to the physicians by females in general. Analysis of all encounters by age and sex in primary healthcare centres in Iceland during 2005 , which shows a pattern similar to that observed in our data, gives support to this explanation [21].

People aged 15-30 years were probably at increased risk of acquiring the pandemic virus during July to September 2009 due to risky behaviour with frequent travel abroad and spending weekends at crowded outdoor festivals in Iceland. The age distribution in Iceland is in accordance with a recently published serological study from England that showed pre-existing antibodies in older age groups that protected against infection [22].

There are uncertainties in our estimate of the true number of pandemic influenza cases in the community. 
The number of samples sent for virological analysis varied over time and it is possible that some samples were false negative. The exact proportion of patients with ILI in community who contacted healthcare was unknown and may have varied between regions and by sex and age group. Multiplying each reported ILI case by 10 should give a rough estimate of the number of cases in the community. Although the care-seeking behaviour for influenza in Iceland has not been studied, an estimate of 1 in 10 seeking care is supported by a recent serological study [22]. It may be possible to estimate the proportion of infected individuals seeking healthcare more accurately using a detailed disease transmission model, but such analysis is beyond the scope of this paper and we leave this for future study.

A small study, based on a questionnaire, carried out in the Akureyri municipality in northern Iceland in mid-November 2009 on the true incidence of ILI in the community showed a $22 \%$ cumulative attack rate (unpublished data), supporting the outcome of the simple model described in this study with regard to age, sex and timing of the epidemic curve by onset of illness. We therefore estimated that the percentage of symptomatic people infected in the community ranged from $10 \%$ to $22 \%$. Estimates from other countries for the 2009 pandemic also concluded that the percentage of people infected with the pandemic virus was less than $30 \%$ of the population $[18,22]$.

There are limitations to our ILI surveillance system. It was developed just in time for the pandemic, had not been adequately tested and baseline data for ILI had not been established. It is possible that physicians were affected by the introduction of a new reporting system and the ongoing pandemic in their clinical assessment. However, the ARI surveillance data do not support this hypothesis. They showed that physicians used ICD-10 codes for ARI when influenza was not suspected. The number of ARI cases peaked in week 41, which probably reflects the increase in illness caused by respiratory viruses other than influenza and/or the pandemic virus in cases with mild symptoms. In our study, ARI was used for quality assurance but further development is intended to enable timely and accurate ARI surveillance.

Our analysis of the data from elementary schools accounts for school absenteeism in number of days absent. The analysis of school absenteeism needs to be developed further with age-specific data on the number of children absent in each school. It is a novel method to estimate the number of children with ILI in the community for every ILI case registered in the healthcare system. It also enables assessment of the socio-economic impact of parents caring for sick children at home and ultimately enables real-time monitoring of local or widespread outbreaks in schools.

The pandemic virus circulated in the community in Iceland during summer and autumn. Elementary schools started in late August, with moderate spread of ILI in schoolchildren during September. But it remains unclear why a large outbreak occurred in October in children attending these schools, rather than in early September, immediately after the schools started.

Our study shows how the sudden need for improved surveillance during the pandemic led to rapid improvements in data collection. However, it is, of course, preferable to have a system in place when pandemics hit. Retrospective data were not collected during the pandemic for two main reasons: firstly, the amount of data would have overloaded both the database and the electronic reporting system and secondly, there was no time to check the validity of the older data and compare with the real-time data during the pandemic. Retrospective data will be collected and a baseline for ILI will be established in future work.

Using the same software for patient records and for surveillance provides a unique opportunity for realtime surveillance and risk assessment. No human input is needed to report the cases, which secures the sustainability of the system and improves the data delivery, compared with the old paper-based reporting system, with regard to the completeness and the timeliness of the data. The data are delivered when the physician has confirmed his record for the patient visit in the electronic patient journal, which can be a problem if physicians postpone their confirmation for weeks, months or even longer. The physicians were, however, constantly reminded during the pandemic to confirm the patient record, but this may need improvements.

The surveillance system established during the pandemic has replaced the older paper-based reporting system for ILI and will be expanded and improved to replace the current system of surveillance of all other notifiable diseases, thus eliminating all paper-based reporting, Changes to the system can be done rapidly, enabling real time surveillance of new and emerging diseases and syndromes that may appear in hospitals and primary healthcare centres in Iceland.

\section{Acknowledgements}

The authors thank Ingi Steinar Ingason, Auður Jóhannesdóttir, Júlíana Jóna Hédinsdóttir, Kristinn Jónsson and our co-workers at EMR for their support and solutions during the pandemic and development of the automatic reporting system for ILI.

\section{References}

1. World Health Organization (WHO). Influenza-like illness in the United States and Mexico. Geneva:WHO; 24 Apr 2009. Available from: http://www.who.int/csr/don/2009_04_24/en/ index.html

2. World Health Organization (WHO). Swine influenza. Statement by WHO Director-General, Dr Margaret Chan. Geneva:WHO; 27 Apr 2009. Available from: http://www.who.int/mediacentre/ news/statements/2009/h1n1_20090427/en/index.html 
3. World Health Organization (WHO). Influenza $A\left(\mathrm{H}_{1} \mathrm{~N}_{1}\right)$. Statement by WHO Director-General, Dr Margaret Chan. Geneva:WHO; 29 Apr 2009. Available from: http://www.who. int/mediacentre/news/statements/2009/h1n1_20090429/en/ index.html

4. Statens Serum Institut (SSI). European monitoring of excess mortality for public health action. Copenhagen: SSI; [Accessed 20 Sep 2010]. Available from: http://www.euromomo.eu/index. html

5. Mazick A, Gergonne B, Wuillaume F, Danis K, Vantarakis A, Uphoff $\mathrm{H}$, et al. Higher all-cause mortality in children during autumn 2009 compared with the three previous years: pooled results from eight European countries. Euro Surveill. 2010;15(5):pii=19480. Available from: http://www. eurosurveillance.org/ViewArticle.aspx?Articleld =19480

6. Schmidt WP, Pebody R, Mangtani P. School absence data for influenza surveillance: a pilot study in the United Kingdom. Euro Surveill. 2010;15(3):pii=19467. Available from: http:// www.eurosurveillance.org/ViewArticle.aspx?Articleld $=19467$

7. Heffernan R, Mostashari F, Das D, Karpati A, Kulldorff M, Weiss D. Syndromic surveillance in public health practice, New York City. Emerg Infect Dis. 2004;10(5):858-64.

8. Lajous M, Danon L, López-Ridaura R, Astley CM, Miller JC, Dowell SF, et al. Mobile messaging as surveillance tool during pandemic $\left(\mathrm{H}_{1} \mathrm{~N}_{1}\right)$ 2009, Mexico. Emerg Infect Dis. 2010;16(9):1488-9.

9. Mentor. [Internet]. Reykjavík:Mentor ehf. [Accessed 9 Dec 2010]. Available from: https://mentor.is/mentor_website/ home/Default.aspx

10. EMR. [Internet]. Reykjavík: EMR. [Accessed 9 Dec 2010]. Available from: http://www.emr.is/

11. World Health Organization (WHO)/German Institute of Medical Documentation and Information (DIMDI).. International Statistical Classification of Diseases and Related Health Problems 10th Revision. Version for 2007. Geneva:WHO/DIMDI; [Accessed 20 Sep 2010]. Available from: http://apps.who.int/ classifications/apps/icd/icd10online/

12. Okkes I, Jamoulle M, Lamberts H, Bentzen N. ICPC-2-E. The electronic version of ICPC-2. Differences with the printed version and the consequences. Fam Pract. 2000;17(2):101-7.

13. Official Journal of the European Union 18.6.2008 L 159/65. Commission Decision of 28 April 2008 amending Decision $2002 / 253 / E C$ laying down case definitions for reporting communicable diseases to the Community network under Decision No 2119/98/EC of the European Parliament and of the Council (reference number C(2008) 1589) 2008/427/ EC. Available from: http://eur-lex.europa.eu/LexUriServ/ LexUriServ.do?uri=0J:L:2008:159:0046:0090:EN:PDF

14. Sigmundsdottir G, Gudnason T, Briem H. Vöktun nýrrar inflúensu $A\left(\mathrm{H}_{1} \mathrm{~N}_{1}\right)$ veiru. [Monitoring the new influenza $A\left(\mathrm{H}_{1} \mathrm{~N}_{1}\right)$ virus]. Laeknabladid. 2009;95(6):466-7. Available from: http:// www.laeknabladid.is/media/tolublod/1424/PDF/uo8.pdf

15. World Health Organization (WHO). CDC protocol of realtime RTPCR for influenza $A\left(\mathrm{H}_{1} \mathrm{~N}_{1}\right)$. Geneva:WHO; 6 Oct 2009. Available from: http://www.who.int/csr/ resources/publications/swineflu/CDCRealtimeRTPCR SwineH1Assay-2009_20090430.pdf

16. Fleming D, Zambon M, Bartelds A I. Population estimates of persons presenting to general practitioners with influenza-like illness, 1987-96: a study of the demography of influenza-like illness in sentinel practice networks in England and Wales, and in The Netherlands. Epidemiol Infect. 2000;124(2): 245-53.

17. Hall IM, Gani R, Hughes HE, Leach S. Real-time epidemic forecasting for pandemic influenza. Epidemiol Infect. 2007; 135(3):372-85.

18. Health Protection Agency (HPA). Method used to estimate new pandemic $\left(\mathrm{H}_{1} \mathrm{~N}_{1}\right) 2009$ influenza cases in England in the week 3 August to 9 August 2009. London:HPA; 12 Aug 2009. Available from: http://www.hpa.org.uk/web/HPAwebFile/ HPAweb_C/1250150839845

19. Paget J, Marquet R, Meijer A, van der Velden K. Influenza activity in Europe during eight seasons (1999-2007): an evaluation of the indicators used to measure activity and an assessment of the timing, length and course of peak activity (spread) across Europe. BMC Infect Dis. 2007;7:141.

20. Toal M, Agyeman-Duah K, Schwenk A, Yoong W. Swine flu and pregnancy. J Obstet Gynaecol. 2010;30(2):97-100.

21. Directorate of Health in Iceland. Samskipti vid heilsugæslustöəvar. [Contacts with the primary health care]. 4 Jul 2007. Available from: http://www.landlaeknir.is/ Pages $/ 1055$ ? NewsID $=1680$

22. Miller E, Hoschler K, Hardelid P, Andrews N, Stanford E, Zambon M. Incidence of 2009 pandemic influenza $\mathrm{A} \mathrm{H}_{1} \mathrm{~N}_{1}$ infection in England: a cross-sectional serological study. Lancet. 2010;375(9720):1100 - 8. 